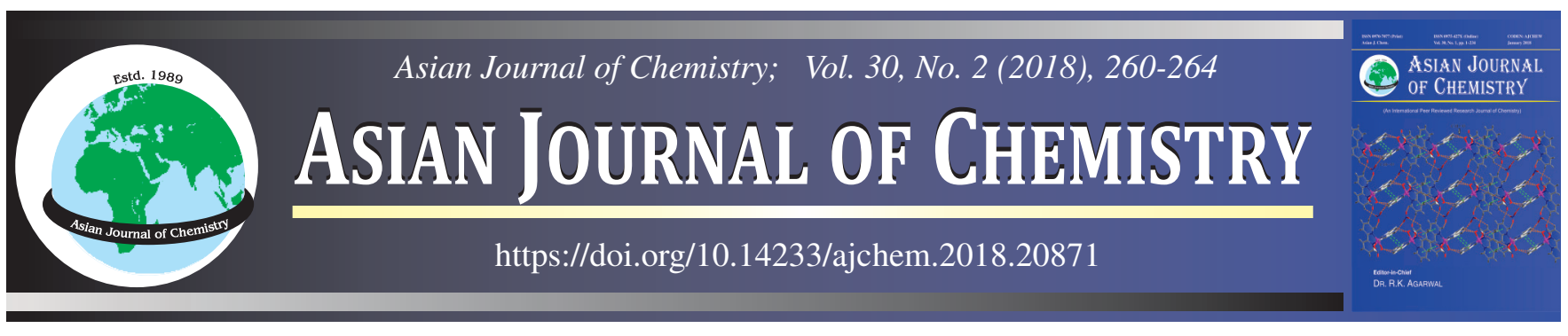

\title{
Synthesis and Structural Analysis of Persuasive Antibacterial Agents and Enzyme Inhibitors Derived from 5-(1-(4-Tosyl)piperidin-4-yl)-1,3,4-oxadiazol-2-thiol
}

Aziz-ur-Rehman ${ }^{1, *}$, Almas Sattar ${ }^{1}$, Muhammad Athar Abbasi $^{1}$, Sabahat Z. Siddiqui ${ }^{1}$, M. Arslan Raza ${ }^{1}$, Muhammad Ashraf ${ }^{2}$, Munibaanum Nazir $^{2}$ and Syed Adnan Ali Shah ${ }^{3}$

${ }^{1}$ Department of Chemistry, Government College University, Lahore-54000, Pakistan

${ }^{2}$ Department of Biochemistry and Biotechnology, The Islamia University of Bahawalpur, Bahawalpur-63100, Pakistan

${ }^{3}$ Faculty of Pharmacy and Atta-ur-Rahman Institute for Natural Products Discovery (AuRIns), Universiti Teknologi MARA, Puncak Alam Campus, 42300 Bandar Puncak Alam, Selangor Darul Ehsan, Malaysia

*Corresponding author: Tel: +92 42111000010 Ext. 450; E-mail: azizryk@yahoo.com

Due to outstanding biological activities of 1,3,4-oxadiazole, a series of $S$-substituted derivatives of 5-[1-(4-tosyl)piperidin-4-yl]-1,3,4oxadiazol-2-thiol (5a-f) was synthesized. The reaction of $p$-toluene sulfonyl chloride (a) with ethyl isonepacotate (b) produced ethyl 1(4-tosyl)piperidin-4-carboxylate (1) which was successively converted to 1-(4-tosyl)piperidin-4-carbohydrazide (2) by hydrazine and 5[1-(4-tosyl)piperidin-4-yl]-1,3,4-oxadiazol-2-thiol (3) by $\mathrm{CS}_{2}$ in the presence of $\mathrm{KOH}$. The aimed compounds (5a-f) were synthesized by the reaction of compound $\mathbf{3}$ with different electrophiles in DMF using lithium hydride as catalyst. The structural confirmation was done by IR, ${ }^{1} \mathrm{H}$ NMR \& EI-MS spectral analysis. The synthesized compounds were screened against $\alpha$-glucosidase enzyme and five Gram bacterial strains.

Keywords: 1,3,4-Oxadiazole, $\alpha$-Glucosidase, Antibacterial activity, Ethyl isonipecotate, Toluenesulfonylchloride.

\section{INTRODUCTION}

Oxadiazoles specially 1,3,4-oxadiazole has gained huge concern which is evident by the literature review of previous fifteen years, during this era more than 2500 publications have been published [1].1,3,4-Oxadiazole moiety containing compounds have diverse range of biological activities [2] including ulcerogenic [3], hypolipidemic [4], cytotoxic [5], anesthetic [6], anti-inflammatory [7], vasodialatory [8], anticonvulsant [9], analgesic [10], antifungal [11], enzyme inhibition [12] and antimicrobial [13]. Among the molecules containing 1,3,4oxadiazole, the derivatives of 1,3,4-oxadiazoles-2-thiones also called as mercapto-oxadiazoles have strong antimicrobial [14] and enzyme inhibition activities [15].

Considering the potent antimicrobial and enzyme inhibition activities of mercapto-oxadiazole, some of its derivatives were synthesized and screened against microbes along with some enzymes to evaluate their biological potential.

\section{EXPERIMENTAL}

Griffin and George melting point apparatus was used to determine the melting points of synthesized compounds in open capillary tube and were not corrected. Thin layer chromatography (TLC) on pre-coated silica gel G-25-UV $\mathrm{U}_{254}$ plates was carried out to confirm purity of the synthesized compounds. The TLC for each compound was developed by using appropriate polarity solvent systems of $n$-hexane and ethyl acetate. All compounds gave single spot on TLC. Jasco320-A spectrometer was used to obtain I.R spectra (wavenumber in $\mathrm{cm}^{-1}$ ) of synthesized compounds with the help of $\mathrm{KBr}$ pellets method. Bruker spectrometer operating at $400 \mathrm{MHz}$ was used to obtain NMR spectra of synthesized compounds in $\mathrm{CDCl}_{3}$ solvent, chemical shifts are given in ppm. JMS-HX-110 spectrometer, with a data system was used to obtain mass spectra (EIMS).

Preparation of ethyl 1-(4-tosyl)piperidin-4-carboxylate (1): Ethyl isonipecotate (a; $10 \mathrm{~mL}, 64.945 \mathrm{mmol})$ was poured in $35 \mathrm{~mL}$ distilled water in a $250 \mathrm{~mL}$ round bottom flask. p-Toluene sulfonyl chloride (b; $7.825 \mathrm{~g}, 64.945 \mathrm{mmol}$ ) was added pinch by pinch in about 20-25 min into the round bottom flask containing suspension of ethyl isonipecotate in water. The $\mathrm{pH}$ of the solution was maintained at 9.0 by basic aqueous solution of $5 \% \mathrm{Na}_{2} \mathrm{CO}_{3}$. The reaction was stirred for 2-3 $\mathrm{h}$. The progress and completion of reaction was monitored with the help of TLC. At the completion of reaction the $\mathrm{pH}$ of reaction mixture was adjusted using $\mathrm{HCl}(2 \mathrm{~mL}, 11 \mathrm{M})$ to 6 . 
The reaction was left for about $15 \mathrm{~min}$. The precipitates of anticipated compound $\mathbf{1}$ were filtered and washed using cold distilled water.

Preparation of 1-(4-tosyl)piperidin-4-carbohydrazide (2): Ethyl 1-(4-tosyl)piperidin-4-carboxylate (1; $5.0 \mathrm{~g}, 16.07$ $\mathrm{mmol}$ ) was dissolved in $25 \mathrm{~mL}$ of methanol in $250 \mathrm{~mL}$ round bottom flask. Hydrazine hydrate $(80 \%, 15 \mathrm{~mL})$ was poured slowly to reaction mixture and refluxed for 3-4 h and reaction progress was monitored by TLC. The white crystals of aimed compound $\mathbf{2}$ were obtained by evaporating extra solvent and adding excess of distilled water. The product was collected through filtration, washed with cold distilled water and dried for further use.

Preparation of 5-[1-(4-tosyl)piperidin-4-yl]-1,3,4oxadiazol-2-thiol (3): 1-(4-Tosyl)piperidin-4-carbohydrazide $(2 ; 4.0 \mathrm{~g}, 13.46 \mathrm{mmol})$ was dissolved in methanol $(25 \mathrm{~mL})$ in a $250 \mathrm{~mL}$ round bottom flask. Potassium hydroxide $(0.75 \mathrm{~g}$, $13.46 \mathrm{mmol})$ and carbon disulfide $(1.3 \mathrm{~mL}, 26.92 \mathrm{mmol})$ was added to the reaction mixture. Reaction mixture was refluxed for 5-6 $\mathrm{h}$ and monitored to verify completion by TLC. At the completion of reaction, chilled distilled water $(50 \mathrm{~mL})$ was added to the reaction mixture and then acidified to $\mathrm{pH} 2-3$ with dilute hydrochloric acid to get the solid precipitates. Off white precipitates of product 3 were filtered, washed with cold distilled water and dried.

General procedure for the synthesis of $S$-substituted derivatives of 3 (5a-f): 5-[1-(4-Tosyl)piperidin-4-yl]-1,3,4oxadiazol-2-thiol $(3 ; 0.0339 \mathrm{~g}, 0.1 \mathrm{mmol})$ was taken in round bottom flask (50 mL). $N, N$-Dimethyl formamide (DMF, $10 \mathrm{~mL}$ ) was added to dissolve compound $\mathbf{3}$ followed by the addition of sodium hydride $(0.0024 \mathrm{~g}, 0.1 \mathrm{mmol})$ at room temperature and stirred for $0.5 \mathrm{~h}$. Then alkyl/aralkyl halides (4a-f), were added in an equimolar ratio to $\mathbf{3}$. The mixture was stirred for 3-4 h. The progress of reaction was monitored with TLC till single spot. Distilled water was added to reaction mixture and products (5a-f) were recovered by filtration followed by washing and drying.

Ethyl 1-(4-tosyl)piperidin-4-carboxylate (1): White amorphous solid; Yield: $89 \%$; m.p.: 70-72 ${ }^{\circ} \mathrm{C}$; m.f.: $\mathrm{C}_{15} \mathrm{H}_{21} \mathrm{NO}_{4} \mathrm{~S}$; m.w.: 311 ; IR $\left(\mathrm{KBr}, v_{\max }, \mathrm{cm}^{-1}\right)$ : 3067 (C-H stretching of aromatic ring), 1732 ( $\mathrm{C}=\mathrm{O}$ stretching), 1531 ( $\mathrm{C}=\mathrm{C}$ aromatic stretching), 1335 (-SO $\mathrm{SO}_{2}$-stretching), 1079 (C-O bond stretching); ${ }^{1} \mathrm{H} \mathrm{NMR}$ (400 MHz, $\mathrm{CDCl}_{3}, \delta, \mathrm{ppm}$ ): 7.62 (d, $J=8.0 \mathrm{~Hz}, 2 \mathrm{H}, \mathrm{H}-2 "$ \& H-6"), 7.32 (d, J = 8.0 Hz, 2H, H-3" \& H-5"), 3.98 (q, J = 7.2 $\mathrm{Hz}, 2 \mathrm{H}, \mathrm{O}-\mathrm{CH}_{2}$ ), 3.71-3.68 (m, 2H, $\left.\mathrm{H}_{e^{-}} 2^{\prime} \& \mathrm{H}_{e}-6^{\prime}\right)$, 2.73-2.62 (m, 1H, H-4'), 2.54-2.48 (m, 2H, $\left.\mathrm{H}_{a}-2^{\prime} \& \mathrm{H}_{a}-6^{\prime}\right), 2.42$ (s, 3H, $\left.\mathrm{CH}_{3}-4^{\prime \prime}\right), 2.10-2.08$ (m, 2H, $\left.\mathrm{H}_{e}-3^{\prime} \& \mathrm{H}_{e}-5^{\prime}\right), 1.60-1.86$ (m, 2H, $\left.\mathrm{H}_{a^{-}} 3^{\prime} \& \mathrm{H}_{a^{-}} 5^{\prime}\right), 1.15\left(\mathrm{t}, J=7.2 \mathrm{~Hz}, \mathrm{CH}_{3}\right)$; $\operatorname{EIMS}(\mathrm{m} / \mathrm{z}): 311$ $[\mathrm{M}]^{+}, 266\left[\mathrm{C}_{13} \mathrm{H}_{16} \mathrm{NO}_{3} \mathrm{~S}\right]^{+}, 238\left[\mathrm{C}_{12} \mathrm{H}_{16} \mathrm{NO}_{2} \mathrm{~S}\right]^{+}, 184\left[\mathrm{C}_{8} \mathrm{H}_{10} \mathrm{NO}_{2} \mathrm{~S}^{+}\right.$, $170\left[\mathrm{C}_{7} \mathrm{H}_{8} \mathrm{NO}_{2} \mathrm{~S}\right]^{+}, 155\left[\mathrm{C}_{7} \mathrm{H}_{7} \mathrm{O}_{2} \mathrm{~S}\right]^{+}, 91\left[\mathrm{C}_{7} \mathrm{H}_{7}\right]^{+}$.

1-(4-Tosyl)piperidin-4-carbohydrazide (2): White crystalline solid; Yield: $91 \%$; m.p.: $128-130{ }^{\circ} \mathrm{C}$; m.f.: $\mathrm{C}_{13} \mathrm{H}_{19} \mathrm{~N}_{3} \mathrm{O}_{3} \mathrm{~S}$; m.w.: 297; IR (KBr, $\left.v_{\max }, \mathrm{cm}^{-1}\right)$ : 3348 (N-H stretching), 3063 (C-H stretching of aromatic ring), 1682 (C=O stretching), 1534 $(\mathrm{C}=\mathrm{C}$ aromatic stretching $), 1339\left(-\mathrm{SO}_{2}\right.$ - stretching $) ;{ }^{1} \mathrm{H} \mathrm{NMR}$ (400 MHz, $\left.\mathrm{CDCl}_{3}, \delta, \mathrm{ppm}\right): 7.61(\mathrm{~d}, J=8.0 \mathrm{~Hz}, 2 \mathrm{H}, \mathrm{H}-2 "$ \& H-6"), 7.33 (d, J=8.0 Hz, 2H, H-3" \& H-5"), 3.72-3.69 (m, $\left.2 \mathrm{H}, \mathrm{H}_{e}-2^{\prime} \& \mathrm{H}_{e}-6^{\prime}\right), 2.73-2.62\left(\mathrm{~m}, 1 \mathrm{H}, \mathrm{H}-44^{\prime}\right), 2.53-2.49$ (m, $\left.2 \mathrm{H}, \mathrm{H}_{a}-2^{\prime} \& \mathrm{H}_{a}-6 '\right), 2.42$ (s, 3H, $\left.\mathrm{CH}_{3}-4^{\prime \prime}\right), 2.12-2.10$ (m, 2H, $\left.\mathrm{H}_{e^{-}} 3^{\prime} \& \mathrm{H}_{e^{-}} 5^{\prime}\right)$, 1.58-1.84 (m, 2H, $\left.\mathrm{H}_{a^{-}} 3^{\prime} \& \mathrm{H}_{a^{-}} 5^{\prime}\right)$; $\operatorname{EIMS}(\mathrm{m} / \mathrm{z})$ : $297[\mathrm{M}]^{+}, 266\left[\mathrm{C}_{13} \mathrm{H}_{16} \mathrm{NO}_{3} \mathrm{~S}\right]^{+}, 238\left[\mathrm{C}_{12} \mathrm{H}_{16} \mathrm{NO}_{2} \mathrm{~S}\right]^{+}, 184$ $\left[\mathrm{C}_{8} \mathrm{H}_{10} \mathrm{NO}_{2} \mathrm{~S}\right]^{+}, 170\left[\mathrm{C}_{7} \mathrm{H}_{8} \mathrm{NO}_{2} \mathrm{~S}^{+}, 155\left[\mathrm{C}_{7} \mathrm{H}_{7} \mathrm{O}_{2} \mathrm{~S}\right]^{+}, 91\left[\mathrm{C}_{7} \mathrm{H}_{7}\right]^{+}\right.$.

5-[1-(4-Tosyl)piperidin-4-yl]-1,3,4-oxadiazol-2-thiol (3): White amorphous solid; Yield: $87 \%$; m.p.: 230-233 ${ }^{\circ} \mathrm{C}$; m.f.: $\mathrm{C}_{14} \mathrm{H}_{17} \mathrm{~N}_{3} \mathrm{O}_{3} \mathrm{~S}_{2}$; m.w.: 339; IR $\left(\mathrm{KBr}, \mathrm{v}_{\max }, \mathrm{cm}^{-1}\right)$ : $3067(\mathrm{C}-\mathrm{H}$ stretching of aromatic ring), 2522 ( $\mathrm{S}-\mathrm{H}$ bond stretching), 1641 $(\mathrm{C}=\mathrm{N}$ stretching of oxadiazole ring $), 1541(\mathrm{C}=\mathrm{C}$ aromatic stretching), 1345 (-SO $2^{-}$stretching), $1249 \& 1079$ (C-O-C bond stretching); ${ }^{1} \mathrm{H} \mathrm{NMR}\left(400 \mathrm{MHz}, \mathrm{CDCl}_{3}, \delta, \mathrm{ppm}\right): 7.63$ (d, $J=$ $8.0 \mathrm{~Hz}, 2 \mathrm{H}, \mathrm{H}-2 "$ \& H-6"), 7.32 (d, $J=8.0 \mathrm{~Hz}, 2 \mathrm{H}, \mathrm{H}-3 "$ \& H5"), 3.71-3.68 (m, 2H, H $\left.e_{e} 2^{\prime} \& \mathrm{H}_{e}-6^{\prime}\right), 2.74-2.63$ (m, 1H, H$\left.4^{\prime}\right)$, 2.54-2.48 (m, 2H, $\left.\mathrm{H}_{a^{-}} 2^{\prime} \& \mathrm{H}_{a^{-}} 6^{\prime}\right), 2.42$ (s, 3H, $\left.\mathrm{CH}_{3}-4^{\prime \prime}\right)$, 2.10-2.08 (m, 2H, $\left.\mathrm{H}_{e}-3^{\prime} \& \mathrm{H}_{e^{-}} 5^{\prime}\right), 1.59-1.85$ (m, 2H, $\mathrm{H}_{a^{-}} 3^{\prime}$ \& $\left.\mathrm{H}_{a^{-}} 5^{\prime}\right)$; $\operatorname{EIMS}(\mathrm{m} / \mathrm{z}): 339[\mathrm{M}]^{+}, 266\left[\mathrm{C}_{13} \mathrm{H}_{16} \mathrm{NO}_{3} \mathrm{~S}\right]^{+}, 238$ $\left[\mathrm{C}_{12} \mathrm{H}_{16} \mathrm{NO}_{2} \mathrm{~S}\right]^{+}, 184\left[\mathrm{C}_{8} \mathrm{H}_{10} \mathrm{NO}_{2} \mathrm{~S}\right]^{+}, 170\left[\mathrm{C}_{7} \mathrm{H}_{8} \mathrm{NO}_{2} \mathrm{~S}\right]^{+}, 155$ $\left[\mathrm{C}_{7} \mathrm{H}_{7} \mathrm{O}_{2} \mathrm{~S}\right]^{+}, 91\left[\mathrm{C}_{7} \mathrm{H}_{7}\right]^{+}$.

4-[2-(Allylthio)-1,3,4-oxadiazol-5-yl]-1-(4-tosyl)piperidine (5a): Light yellow amorphous solid; Yield: $75 \%$; m.p.: 115-117 ${ }^{\circ} \mathrm{C}$; m.f.: $\mathrm{C}_{17} \mathrm{H}_{21} \mathrm{~N}_{3} \mathrm{O}_{3} \mathrm{~S}_{2}$; m.w.: 379; IR $\left(\mathrm{KBr}, v_{\max }, \mathrm{cm}^{-1}\right)$ : 3055 (C-H stretching of aromatic ring), 1648 (C=N stretching of oxadiazole ring), $1677(\mathrm{C}=\mathrm{C}$ stretching of allyl), $1537(\mathrm{C}=\mathrm{C}$ aromatic stretching), 1354 (-SO ${ }_{2}$ - stretching), $1257 \& 1076$ (C-O-C bond stretching); ${ }^{1} \mathrm{H}$ NMR (400 MHz, $\left.\mathrm{CDCl}_{3}, \delta, \mathrm{ppm}\right)$ : 7.63 (d, $J=8.0 \mathrm{~Hz}, 2 \mathrm{H}, \mathrm{H}-2 " \& \mathrm{H}-6 "), 7.31$ (d, $J=8.0 \mathrm{~Hz}, 2 \mathrm{H}$, H-3" \& H-5"), 5.97-5.88 (m, 1H, H-2"'), 5.33-5.29 (m, 1H, $\left.\mathrm{H}_{\mathrm{a}}-3^{\prime \prime \prime}\right), 5.17-5.12\left(\mathrm{~m}, 1 \mathrm{H}, \mathrm{H}_{\mathrm{b}}-3^{\prime \prime \prime}\right), 3.81$ (d, $J=6.8 \mathrm{~Hz}, 2 \mathrm{H}$, $\left.\mathrm{CH}_{2}-1^{\prime \prime \prime}\right), 2.87-2.81$ (m, 2H, $\left.\mathrm{H}_{e}-2^{\prime} \& \mathrm{H}_{e^{-}} 6^{\prime}\right), 2.86-2.81$ (m, $1 \mathrm{H}$, H-4'), 2.61-2.54 (m, 2H, H $\left.a_{a}-2^{\prime} \& \mathrm{H}_{a}-6^{\prime}\right), 2.42$ (s, 3H, $\left.\mathrm{CH}_{3}-4^{\prime \prime}\right)$, 2.12-2.07 (m, 2H, $\left.\mathrm{H}_{e}-3^{\prime} \& \mathrm{H}_{e^{-}} 5^{\prime}\right), 2.00-1.91$ (m, 2H, $\mathrm{H}_{a}-3^{\prime}$ \& $\left.\mathrm{H}_{a^{-}} 5^{\prime}\right)$;EIMS $(\mathrm{m} / \mathrm{z}): 379[\mathrm{M}]^{+}, 266\left[\mathrm{C}_{13} \mathrm{H}_{16} \mathrm{NO}_{3} \mathrm{~S}^{+}, 238\right.$ $\left[\mathrm{C}_{12} \mathrm{H}_{16} \mathrm{NO}_{2} \mathrm{~S}\right]^{+}, 184\left[\mathrm{C}_{8} \mathrm{H}_{10} \mathrm{NO}_{2} \mathrm{~S}\right]^{+}, 170\left[\mathrm{C}_{7} \mathrm{H}_{8} \mathrm{NO}_{2} \mathrm{~S}\right]^{+}, 155$ $\left[\mathrm{C}_{7} \mathrm{H}_{7} \mathrm{O}_{2} \mathrm{~S}\right]^{+}, 91\left[\mathrm{C}_{7} \mathrm{H}_{7}\right]^{+}, 83\left[\mathrm{C}_{5} \mathrm{H}_{9} \mathrm{~N}\right]^{+}, 41\left[\mathrm{C}_{3} \mathrm{H}_{5}\right]^{+}$.

4-[2-(2-Chloroethylthio)-1,3,4-oxadiazol-5-yl]-1-(4tosyl)piperidine (5b): Light yellow amorphous solid; Yield: $81 \%$; m.p.: 212-214 ${ }^{\circ}$ C; m.f.: $\mathrm{C}_{16} \mathrm{H}_{20} \mathrm{~N}_{3} \mathrm{O}_{3} \mathrm{~S}_{2} \mathrm{Cl}$; m.w.: 401; IR $\left(\mathrm{KBr}, v_{\max }, \mathrm{cm}^{-1}\right): 3062$ (C-H stretching of aromatic ring), $1643(\mathrm{C}=\mathrm{N}$ stretching of oxadiazole ring), $1533(\mathrm{C}=\mathrm{C}$ aromatic stretching), 1335 (-SO ${ }_{2}$ - stretching), $1249 \& 1079$ (C-O-C bond stretching), 713 (C-Cl bond stretching); ${ }^{1} \mathrm{H}$ NMR (400 MHz, $\mathrm{CDCl}_{3}, \delta$, ppm): 7.63 (d, $\left.J=8.0 \mathrm{~Hz}, 2 \mathrm{H}, \mathrm{H}-2 " \& \mathrm{H}-6 "\right), 7.31$ (d, $J=8.0 \mathrm{~Hz}, 2 \mathrm{H}, \mathrm{H}-3 "$ " \& H-5"), 3.81 (t, $J=7.2 \mathrm{~Hz}, 2 \mathrm{H}, \mathrm{H}-$ $2^{\prime \prime \prime)}, 3.53$ (t, J=7.2 Hz, 2H, H-1"'), 3.31-3.26 (m, 2H, $\mathrm{H}_{e}-2$ ' \& $\left.\mathrm{H}_{e^{-}} 6^{\prime}\right), 2.87-2.80$ (m, 1H, H-4'), 2.60-2.54 (m, 2H, $\mathrm{H}_{a^{-}} 2^{\prime} \& \mathrm{H}_{a^{-}}$ 6'), 2.42 (s, 3H, $\left.\mathrm{CH}_{3}-4^{\prime \prime}\right), 2.12-2.08$ (m, 2H, $\left.\mathrm{H}_{e}-3^{\prime} \& \mathrm{H}_{e}-5^{\prime}\right)$, 2.01-1.94 (m, 2H, $\left.\mathrm{H}_{a^{-}} 3^{\prime} \& \mathrm{H}_{a^{-}} 5^{\prime}\right)$; $\operatorname{EIMS}(\mathrm{m} / \mathrm{z}): 403[\mathrm{M}+2]^{+}$, $401[\mathrm{M}]^{+}, 266\left[\mathrm{C}_{13} \mathrm{H}_{16} \mathrm{NO}_{3} \mathrm{~S}\right]^{+}, 238\left[\mathrm{C}_{12} \mathrm{H}_{16} \mathrm{NO}_{2} \mathrm{~S}\right]^{+}, 184$ $\left[\mathrm{C}_{8} \mathrm{H}_{10} \mathrm{NO}_{2} \mathrm{~S}\right]^{+}, 170\left[\mathrm{C}_{7} \mathrm{H}_{8} \mathrm{NO}_{2} \mathrm{~S}^{+}, 155\left[\mathrm{C}_{7} \mathrm{H}_{7} \mathrm{O}_{2} \mathrm{~S}\right]^{+}, 91\left[\mathrm{C}_{7} \mathrm{H}_{7}\right]^{+}\right.$, $83\left[\mathrm{C}_{5} \mathrm{H}_{9} \mathrm{~N}\right]^{+}, 63\left[\mathrm{C}_{2} \mathrm{H}_{4} \mathrm{Cl}\right]^{+}$.

4-[2-(2-Bromoethylthio)-1,3,4-oxadiazol-5-yl]-1-(4tosyl)piperidine (5c): Fluffy white solid; Yield: $81 \%$; m.p.: 223-225 ${ }^{\circ}$ C; m.f.: $\mathrm{C}_{16} \mathrm{H}_{20} \mathrm{~N}_{3} \mathrm{O}_{3} \mathrm{~S}_{2} \mathrm{Br}$; m.w.: 445; IR (KBr, $v_{\max }$, $\left.\mathrm{cm}^{-1}\right)$ : $3061(\mathrm{C}-\mathrm{H}$ stretching of aromatic ring), $1642(\mathrm{C}=\mathrm{N}$ stretching of oxadiazole ring), $1529(\mathrm{C}=\mathrm{C}$ aromatic stretching), 1331 (- $\mathrm{SO}_{2}$ - stretching), $1247 \& 1077$ (C-O-C bond stretching), 634 (C-Br bond stretching); ${ }^{1} \mathrm{H}$ NMR (400 MHz, $\mathrm{CDCl}_{3}, \delta$, ppm): 7.59 (d, $\left.J=8.0 \mathrm{~Hz}, 2 \mathrm{H}, \mathrm{H}-2^{\prime \prime} \& \mathrm{H}-6 "\right), 7.29$ (d, $J=8.0$ 
Hz, 2H, H-3" \& H-5"), 3.71 (t, J=7.2 Hz, 2H, H-2"'), 3.43 (t, $\left.J=7.2 \mathrm{~Hz}, 2 \mathrm{H}, \mathrm{H}-1^{\prime \prime \prime}\right), 3.32-3.27$ (m, 2H, $\left.\mathrm{H}_{e^{-}} 2^{\prime} \& \mathrm{H}_{e^{-}}-6 '\right), 2.87-$ 2.80 (m, 1H, H-4'), 2.60-2.54 (m, 2H, $\left.\mathrm{H}_{a^{-}} 2^{\prime} \& \mathrm{H}_{a^{-}} 6^{\prime}\right), 2.42$ (s, $\left.3 \mathrm{H}, \mathrm{CH}_{3}-4^{\prime \prime}\right)$, 2.09-2.07 (m, 2H, $\mathrm{H}_{e}-3^{\prime} \& \mathrm{H}_{e}-5^{\prime}$ ), 2.00-1.93 (m, $\left.2 \mathrm{H}, \mathrm{H}_{a^{-}} 3^{\prime} \& \mathrm{H}_{a^{-}} 5^{\prime}\right)$; EIMS (m/z): $447[\mathrm{M}+2]^{+}, 445[\mathrm{M}]^{+}, 266$ $\left[\mathrm{C}_{13} \mathrm{H}_{16} \mathrm{NO}_{3} \mathrm{~S}\right]^{+}, 238\left[\mathrm{C}_{12} \mathrm{H}_{16} \mathrm{NO}_{2} \mathrm{~S}\right]^{+}, 184\left[\mathrm{C}_{8} \mathrm{H}_{10} \mathrm{NO}_{2} \mathrm{~S}\right]^{+}, 170$ $\left[\mathrm{C}_{7} \mathrm{H}_{8} \mathrm{NO}_{2} \mathrm{~S}\right]^{+}, 155\left[\mathrm{C}_{7} \mathrm{H}_{7} \mathrm{O}_{2} \mathrm{~S}\right]^{+}, 107\left[\mathrm{C}_{2} \mathrm{H}_{4} \mathrm{Br}\right]^{+} 91\left[\mathrm{C}_{7} \mathrm{H}_{7}\right]^{+}, 83$ $\left[\mathrm{C}_{5} \mathrm{H}_{9} \mathrm{~N}\right]^{+}$.

4-[2-(2-Phenylethylthio)-1,3,4-oxadiazol-5-yl]-1-(4tosyl)piperidine (5d): Fluffy white solid; Yield: $85 \%$; m.p.: 120-122 ${ }^{\circ} \mathrm{C}$; m.f.: $\mathrm{C}_{22} \mathrm{H}_{25} \mathrm{~N}_{3} \mathrm{O}_{3} \mathrm{~S}_{2}$; m.w.: 443; IR (KBr, $\left.v_{\max }, \mathrm{cm}^{-1}\right)$ : 3046 (C-H stretching of aromatic ring), 1657 (C=N stretching of oxadiazole ring), 1541 ( $\mathrm{C}=\mathrm{C}$ aromatic stretching), 1343 (-SO ${ }_{2}$ - stretching), $1242 \& 1083$ (C-O-C bond stretching); ${ }^{1} \mathrm{H}$ NMR (400 MHz, $\mathrm{CDCl}_{3}, \delta$, ppm): 7.63 (d, $J=8.0 \mathrm{~Hz}, 2 \mathrm{H}, \mathrm{H}-$ 2" \& H-6"), 7.31 (d, J = 7.6 Hz, 2H, H-3" \& H-5"), 7.37-7.25 (m, 5H, H-2"' to H-6"'), 4.39 (t, J=5.6 Hz, 2H, $\mathrm{CH}_{2}-7$ "') $^{\prime}, 3.89$ (t, $\left.J=5.6 \mathrm{~Hz}, 2 \mathrm{H}, \mathrm{CH}_{2}-8^{\prime \prime \prime}\right), 3.66-3.63$ (m, 2H, $\left.\mathrm{H}_{e^{-}} 2^{\prime} \& \mathrm{H}_{e^{-}}-6^{\prime}\right)$, 2.84-2.79 (m, 1H, H-4'), 2.60-2.53 (m, 2H, $\left.\mathrm{H}_{a}-2^{\prime} \& \mathrm{H}_{a^{-}} 6^{\prime}\right)$, 2.42 (s, 3H, $\left.\mathrm{CH}_{3}-4^{\prime \prime}\right), 2.10-2.05$ (m, 2H, $\left.\mathrm{H}_{e}-3^{\prime} \& \mathrm{H}_{e}-5^{\prime}\right), 1.98-$ $1.89\left(\mathrm{~m}, 2 \mathrm{H}, \mathrm{H}_{a^{-}} 3^{\prime} \& \mathrm{H}_{a^{-}} 5^{\prime}\right)$; $\operatorname{EIMS}(\mathrm{m} / \mathrm{z}): 443\left[\mathrm{M}^{+}, 266\right.$ $\left[\mathrm{C}_{13} \mathrm{H}_{16} \mathrm{NO}_{3} \mathrm{~S}\right]^{+}, 238\left[\mathrm{C}_{12} \mathrm{H}_{16} \mathrm{NO}_{2} \mathrm{~S}\right]^{+}, 184\left[\mathrm{C}_{8} \mathrm{H}_{10} \mathrm{NO}_{2} \mathrm{~S}\right]^{+}, 170$ $\left[\mathrm{C}_{7} \mathrm{H}_{8} \mathrm{NO}_{2} \mathrm{~S}^{+}, 155\left[\mathrm{C}_{7} \mathrm{H}_{7} \mathrm{O}_{2} \mathrm{~S}\right]^{+}, 105\left[\mathrm{C}_{8} \mathrm{H}_{9}\right]^{+}, 91\left[\mathrm{C}_{7} \mathrm{H}_{7}\right]^{+}, 83\right.$ $\left[\mathrm{C}_{5} \mathrm{H}_{9} \mathrm{~N}\right]^{+}, 77\left[\mathrm{C}_{6} \mathrm{H}_{5}\right]^{+}, 51\left[\mathrm{C}_{4} \mathrm{H}_{3}\right]^{+}$.

4-[2-(3-Phenylpropylthio)-1,3,4-oxadiazol-5-yl]-1-(4tosyl)piperidine (5e): White crystalline solid; Yield: $85 \%$; m.p.: 115-117 ${ }^{\circ} \mathrm{C}$; m.f.: $\mathrm{C}_{23} \mathrm{H}_{27} \mathrm{~N}_{3} \mathrm{O}_{3} \mathrm{~S}_{2}$; m.w.: 457; IR (KBr, $\left.\mathrm{V}_{\max }, \mathrm{cm}^{-1}\right): 3041(\mathrm{C}-\mathrm{H}$ stretching of aromatic ring $), 1652(\mathrm{C}=\mathrm{N}$ stretching of oxadiazole ring), $1539(\mathrm{C}=\mathrm{C}$ aromatic stretching), 1341 (-SO $\mathrm{S}_{2}$ - stretching), $1241 \& 1082$ (C-O-C bond stretching); ${ }^{1} \mathrm{H}$ NMR (400 MHz, $\mathrm{CDCl}_{3}, \delta$, ppm): 7.63 (d, $J=8.0 \mathrm{~Hz}, 2 \mathrm{H}$, H-2" \& H-6"), 7.31 (d, J = 7.6 Hz, 2H, H-3" \& H-5"), 7.377.25 (m, 5H, H-2"' to H-6"'), 4.45 (t, $\left.J=5.6 \mathrm{~Hz}, 2 \mathrm{H}, \mathrm{CH}_{2}-7^{\prime \prime \prime}\right)$, 3.90 (t, $\left.J=5.6 \mathrm{~Hz}, 2 \mathrm{H}, \mathrm{CH}_{2}-9^{\prime \prime \prime}\right), 3.79-3.76$ (m, 2H, $\left.\mathrm{CH}_{2}-8^{\prime \prime \prime}\right)$, 3.66-3.63 (m, 2H, $\left.\mathrm{H}_{e^{-}} 2^{\prime} \& \mathrm{H}_{e^{-}}-6^{\prime}\right), 2.84-2.79$ (m, 1H, H-4'), 2.60-2.53 (m, 2H, $\left.\mathrm{H}_{a}-2^{\prime} \& \mathrm{H}_{a}-6^{\prime}\right), 2.42$ (s, 3H, $\left.\mathrm{CH}_{3}-4^{\prime \prime}\right), 2.10-$ 2.05 (m, 2H, $\left.\mathrm{H}_{e^{-}} 3^{\prime} \& \mathrm{H}_{e^{-}} 5^{\prime}\right)$, 1.98-1.89 (m, 2H, $\left.\mathrm{H}_{a^{-}} 3^{\prime} \& \mathrm{H}_{a}-5^{\prime}\right)$; EIMS $(\mathrm{m} / z): 457[\mathrm{M}]^{+}, 266\left[\mathrm{C}_{13} \mathrm{H}_{16} \mathrm{NO}_{3} \mathrm{~S}\right]^{+}, 238\left[\mathrm{C}_{12} \mathrm{H}_{16} \mathrm{NO}_{2} \mathrm{~S}\right]^{+}$, $184\left[\mathrm{C}_{8} \mathrm{H}_{10} \mathrm{NO}_{2} \mathrm{~S}\right]^{+}, 170\left[\mathrm{C}_{7} \mathrm{H}_{8} \mathrm{NO}_{2} \mathrm{~S}\right]^{+}, 155\left[\mathrm{C}_{7} \mathrm{H}_{7} \mathrm{O}_{2} \mathrm{~S}\right]^{+}, 119$ $\left[\mathrm{C}_{9} \mathrm{H}_{11}\right]^{+}, 91\left[\mathrm{C}_{7} \mathrm{H}_{7}\right]^{+}, 83\left[\mathrm{C}_{5} \mathrm{H}_{9} \mathrm{~N}\right]^{+}, 77\left[\mathrm{C}_{6} \mathrm{H}_{5}\right]^{+}, 65\left[\mathrm{C}_{5} \mathrm{H}_{5}\right]^{+}, 51$ $\left[\mathrm{C}_{4} \mathrm{H}_{3}\right]^{+}$.

4-[2-((1,3-Dioxolan-2-yl)methylthio)-1,3,4-oxadiazol5-yl]-1-(4-tosyl)piperidine (5f): White powder solid; Yield: $81 \%$; m.p.: 130-132 ${ }^{\circ} \mathrm{C}$; m.f.: $\mathrm{C}_{18} \mathrm{H}_{23} \mathrm{~N}_{3} \mathrm{O}_{5} \mathrm{~S}_{2}$; m.w.: 425; IR
$\left(\mathrm{KBr}, \mathrm{v}_{\max }, \mathrm{cm}^{-1}\right)$ : 3065 (C-H stretching of aromatic ring), 1638 $(\mathrm{C}=\mathrm{N}$ stretching of oxadiazole ring), $1534(\mathrm{C}=\mathrm{C}$ aromatic stretching), 1335 (-SO $\mathrm{SO}_{2}$ - stretching), 1248 \& 1078 (C-O-C bond stretching); ${ }^{1} \mathrm{H} \mathrm{NMR}\left(400 \mathrm{MHz}, \mathrm{CDCl}_{3}, \delta, \mathrm{ppm}\right): 7.63$ (d, $J=$ $8.0 \mathrm{~Hz}, 2 \mathrm{H}, \mathrm{H}-2 "$ \& H-6"), 7.32 (d, J=8.0 Hz, 2H, H-3" \& H5"), 5.10 (t, J=3.60 Hz, 1H, H-5"'), 4.15-4.12 (m, 4H, H-2"' \&H-3"'), 3.71-3.68 (m, 2H, H $e_{e} 2^{\prime} \& \mathrm{H}_{e}-6$ '), 3.33 (d, $J=3.2 \mathrm{~Hz}$, $\left.2 \mathrm{H}, \mathrm{CH}_{2}-6^{\prime \prime \prime}\right), 2.74-2.63$ (m, 1H, H-4') 2.54-2.48 (m, 2H, $\mathrm{H}_{a^{-}} 2^{\prime}$ \& $\left.\mathrm{H}_{a^{-}} 6^{\prime}\right), 2.42$ (s, 3H, $\left.\mathrm{CH}_{3}-4^{\prime \prime}\right), 2.11-2.09$ (m, 2H, $\mathrm{H}_{e^{-}} 3^{\prime} \& \mathrm{H}_{e^{-}}$ 5'), 1.59-1.85 (m, 2H, $\left.\mathrm{H}_{a}-3^{\prime} \& \mathrm{H}_{a}-5^{\prime}\right)$;EIMS ( $\left.\mathrm{m} / \mathrm{z}\right)$ : $425[\mathrm{M}]^{+}$, $266\left[\mathrm{C}_{13} \mathrm{H}_{16} \mathrm{NO}_{3} \mathrm{~S}\right]^{+}, 238\left[\mathrm{C}_{12} \mathrm{H}_{16} \mathrm{NO}_{2} \mathrm{~S}\right]^{+}, 184\left[\mathrm{C}_{8} \mathrm{H}_{10} \mathrm{NO}_{2} \mathrm{~S}\right]^{+}$, $170\left[\mathrm{C}_{7} \mathrm{H}_{8} \mathrm{NO}_{2} \mathrm{~S}^{+}, 155\left[\mathrm{C}_{7} \mathrm{H}_{7} \mathrm{O}_{2} \mathrm{~S}^{+}, 91\left[\mathrm{C}_{7} \mathrm{H}_{7}\right]^{+}, 87\left[\mathrm{C}_{4} \mathrm{H}_{7} \mathrm{O}_{2}\right]^{+}\right.\right.$, $83\left[\mathrm{C}_{5} \mathrm{H}_{9} \mathrm{~N}\right]^{+}, 73\left[\mathrm{C}_{3} \mathrm{H}_{5} \mathrm{O}_{2}\right]^{+}$.

$\alpha$-Glucosidase enzyme inhibition assay: The $\alpha$-glucosidase inhibition activity was assayed according to Chapdelaine et al. [16] method with a little modification. The variation in absorbance before and after the addition of test compound in prepared enzyme solution was noted.

Antibacterial activity assay: The antibacterial activity was performed in sterile 96-wells microplates under aseptic environments. The method is based on the principle that microbial cell number increases as the microbial growth proceeds in a log phase of growth which results in increased absorbance of broth medium [17].

Statistical analysis: All the measurements were performed in triplicate and statistical analysis was obtained from Microsoft Excel 2010. The results are presented as mean \pm SEM. The MIC (minimum inhibitory concentration, measured with suitable dilutions, 5-30 $\mu \mathrm{g} /$ well $)$ and $\mathrm{IC}_{50}$ (50\% inhibitory concentration) were calculated using EZ - Fitz Perrella Scientific Inc. Amherst USA software.

\section{RESULTS AND DISCUSSION}

The aim of present study was to synthesize $S$-substituted derivatives of 1,3,4-oxadiazoles-2-thiones containing piperidine and $p$-toluene sulfonyl moieties along with their biological screening including enzyme inhibition and antibacterial activity. A series of six compounds 5a-f was synthesized according to Scheme-I.

The compound 5a was prepared as light yellow amorphous solid. The molecular formula, $\mathrm{C}_{17} \mathrm{H}_{21} \mathrm{~N}_{3} \mathrm{O}_{3} \mathrm{~S}_{2}$, was established from molecular ion peak at $m / z, 379$ in EIMS and by counting protons from ${ }^{1} \mathrm{H}$ NMR spectrum. The IR spectrum gave peaks at $3055,1648,1677,1537,1354,1257$ and 1076 which were
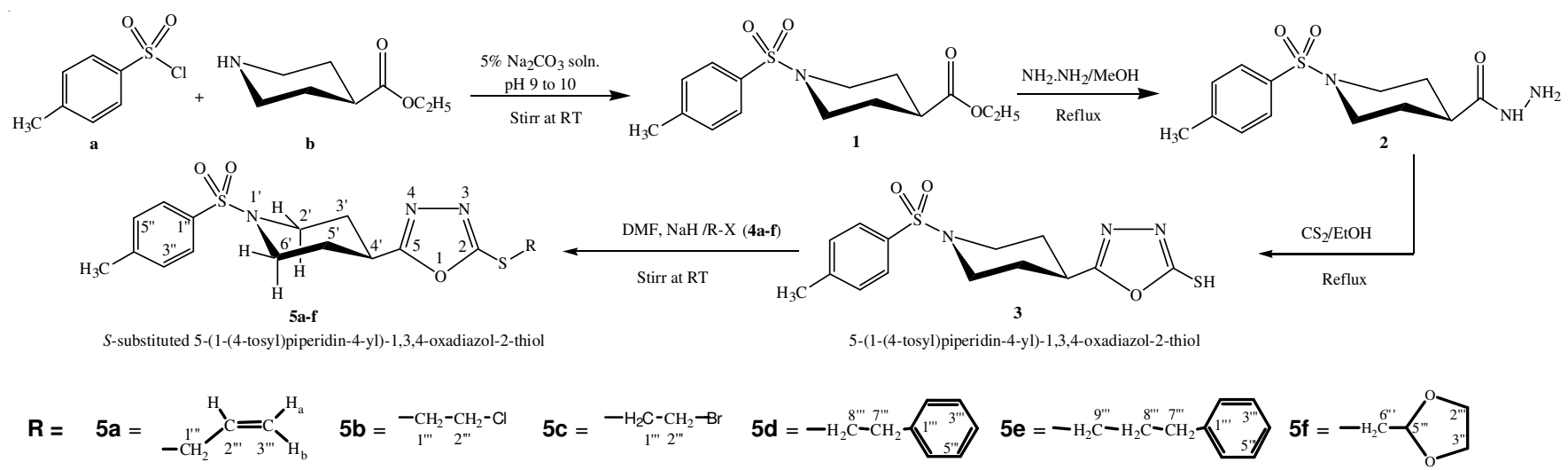

Scheme-I: Outline for the synthesis of $S$-substituted derivatives of 5-(1-(4-tosyl)piperidin-4-yl)-1,3,4-oxadiazol-2-thiol (5a-f) 
assigned to $\mathrm{C}-\mathrm{H}$ (stretching of aromatic ring), $\mathrm{C}=\mathrm{N}$ (stretching of oxadiazole ring), $\mathrm{C}=\mathrm{C}$ (stretching of allyl), $\mathrm{C}=\mathrm{C}$ (aromatic stretching), $-\mathrm{SO}_{2^{-}}$(stretching) and $\mathrm{C}-\mathrm{O}-\mathrm{C}$ (bond stretching of oxadiazole) respectively. In EIMS spectrum, the peak at $\mathrm{m} / \mathrm{z}$ 266 showed the removal of allyl sulfide group along with partial removal of oxadiazole ring from $\mathbf{5 a}$, peak at $\mathrm{m} / \mathrm{z} 155$ showed the presence of $p$-toluenesulfonyl group and peak at $\mathrm{m} / \mathrm{z} 83$ indicated the presence of piperidine moiety. The other prominent fragments are given in Fig. 1. In the aromatic region of ${ }^{1} \mathrm{H}$ NMR signals appeared at $\delta 7.63(\mathrm{~d}, J=8.0 \mathrm{~Hz}, 2 \mathrm{H}, \mathrm{H}-$ 2" \& H-6") and 7.31 (d, J=8.0 Hz, 2H, H-3" \& H-5") indicating the paradisubstituted benzene ring, $p$-toluene sulfony moiety. The signals appearing at $\delta$ 5.97-5.88 (m, 1H, H-2"'), 5.33$5.29\left(\mathrm{~m}, 1 \mathrm{H}, \mathrm{H}_{\mathrm{a}}-3^{\prime \prime \prime}\right), 5.17-5.12\left(\mathrm{~m}, 1 \mathrm{H}, \mathrm{H}_{\mathrm{b}}-3^{\prime \prime \prime}\right)$ and $3.81(\mathrm{~d}$, $\left.J=6.8 \mathrm{~Hz}, 2 \mathrm{H}, \mathrm{CH}_{2}-1^{\prime \prime \prime}\right)$ were assigned to $S$-substituted allyl group. The signals resonating at $\delta 2.87-2.81\left(\mathrm{~m}, 2 \mathrm{H}, \mathrm{H}_{e}-2^{\prime} \&\right.$ $\left.\mathrm{H}_{e}-6^{\prime}\right), 2.86-2.81$ (m, 1H, H-4'), 2.61-2.54 (m, 2H, $\mathrm{H}_{a}-2^{\prime} \&$ $\left.\mathrm{H}_{a^{-}} 6^{\prime}\right), 2.12-2.07\left(\mathrm{~m}, 2 \mathrm{H}, \mathrm{H}_{e^{-}} 3^{\prime} \& \mathrm{H}_{e^{-}} 5^{\prime}\right)$ and 2.00-1.91 (m, $\left.2 \mathrm{H}, \mathrm{H}_{a}-3^{\prime} \& \mathrm{H}_{a}-5^{\prime}\right)$ were assigned to piperidine moiety. The singlet at $\delta 2.42\left(\mathrm{~s}, 3 \mathrm{H}, \mathrm{CH}_{3}-4 "\right)$ indicated the presence of methyl substituent of toluene sulfonyl ring. The structures of other compounds were determined similarly using EIMS, IR and ${ }^{1} \mathrm{H}$ NMR techniques.

Enzyme inhibition activity: The screening of synthesized compounds against $\alpha$-glucosidase enzyme revealed that these compounds are weakly active against $\alpha$-glucosidase. The results are given in Table-1. It is evident from the tabulated data of enzyme inhibition that compound $\mathbf{5} \mathbf{b}$ was the most potent with $\mathrm{IC}_{50}$ value of $190.23 \pm 0.13 \mu \mathrm{M}$ with respect to that of acarbose as $38.25 \pm 0.12 \mu \mathrm{M}$, the reference standard. The compound 5a showed no activity against this enzyme. The somewhat better activity of $\mathbf{5} \mathbf{b}$ can be attributed to chloroethyl substituent attached to sulfur of 5-[1-(4-tosyl)piperidin-4-yl]-1,3,4-oxadiazol-2-thiol. The overall ascending order of inhibition of synthesized compounds is $\mathbf{5} \mathbf{f}<\mathbf{5 e}<\mathbf{5} \mathbf{c}<\mathbf{5 d}<\mathbf{5} \mathbf{b}$.

\begin{tabular}{|c|c|c|}
\hline \multicolumn{3}{|c|}{$\begin{array}{c}\text { TABLE-1 } \\
\text { ENZYME INHIBITION ACTIVITY } \\
\text { AGAINST } \alpha \text {-GLUCOSIDASE ENZYME }\end{array}$} \\
\hline \multirow{2}{*}{ Compound } & \multicolumn{2}{|c|}{$\alpha$-Glucosidase } \\
\hline & Inhibition (\%) at $0.5 \mathrm{mM}$ & $\mathrm{IC}_{50}(\mu \mathrm{M})$ \\
\hline $5 \mathbf{a}$ & $32.15 \pm 0.29$ & - \\
\hline $5 \mathbf{b}$ & $71.34 \pm 0.43$ & $190.23 \pm 0.13$ \\
\hline $5 c$ & $84.43 \pm 0.25$ & $316.49 \pm 0.11$ \\
\hline $5 d$ & $88.15 \pm 0.42$ & $297.92 \pm 0.19$ \\
\hline $5 e$ & $61.33 \pm 0.31$ & $318.57 \pm 0.11$ \\
\hline $5 f$ & $84.46 \pm 0.16$ & $321.43 \pm 0.14$ \\
\hline Control (acarbose) & $92.23 \pm 0.14$ & $38.25 \pm 0.12$ \\
\hline
\end{tabular}

Antibacterial activity: The results of antibacterial activity against Gram-bacteria using ciprofloxacin as reference standard are given in Tables 2 and 3. In this study five bacterial strains were included, two Gram-positive and three Gramnegative. All the compounds showed strong to moderate activity. The compounds $\mathbf{5 b}$ and $\mathbf{5 e}$ showed inhibition of all microbes under consideration. The highest potency of compounds $\mathbf{5 b}$ and $\mathbf{5 e}$ was against Bacillus subtilis and Salmonella typhi, respectively. The compound $\mathbf{5 a}$ inhibited the growth of all microbes under study except that of Staphylococcus aureus. It was also proved most potent against Escherichia coli with MIC value of $9.90 \pm 0.13 \mu \mathrm{M}$ whereas that of standard

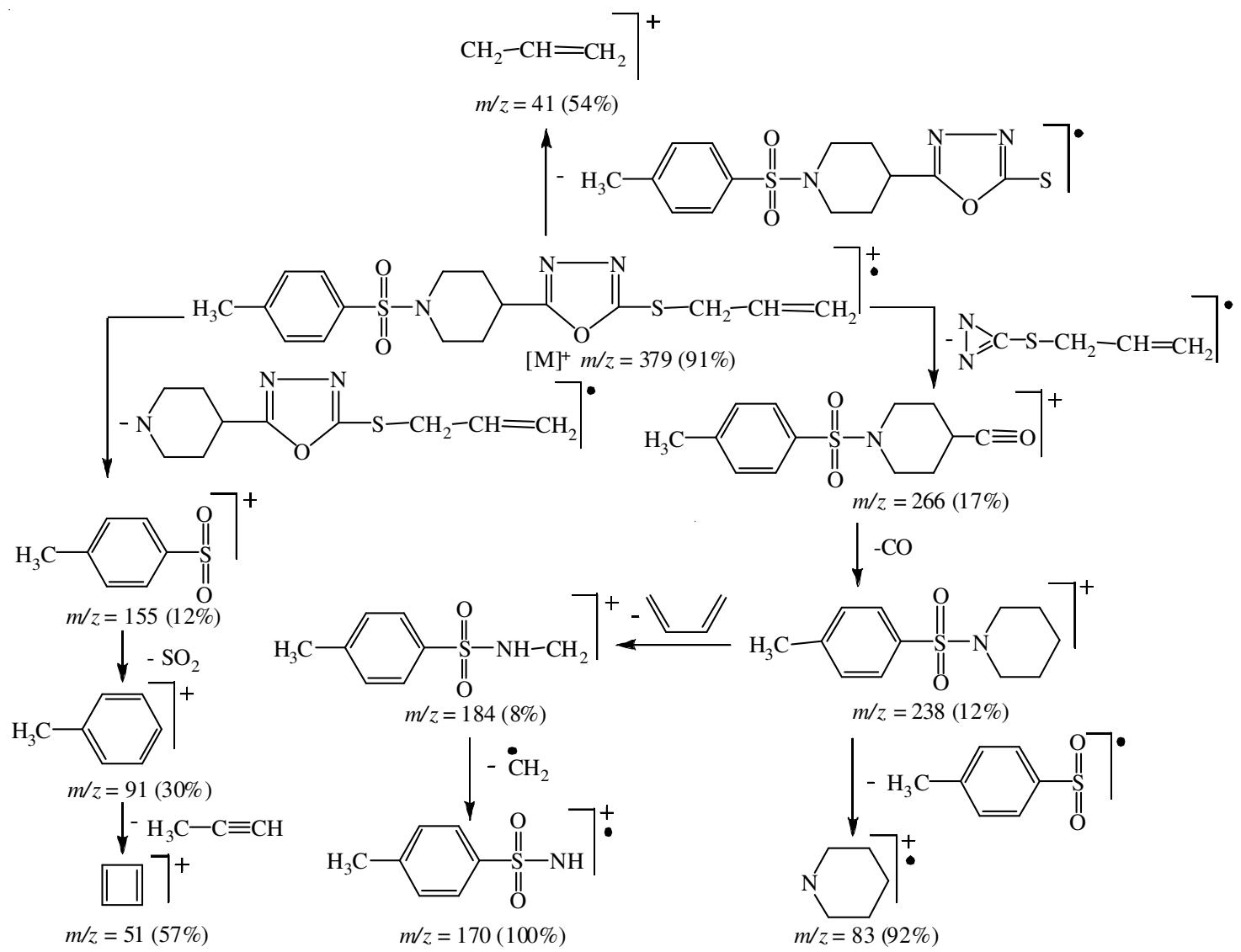

Fig. 1. Mass fragmentation pattern of compound $\mathbf{5 a}$ 


\begin{tabular}{|c|c|c|c|c|c|}
\hline \multirow{2}{*}{ Compounds } & \multicolumn{5}{|c|}{$\begin{array}{c}\text { TABLE-2 } \\
\text { PERCENTAGE INHIBITION OF ANTIBACTERIAL ACTIVITY OF SYNTHESIZED COMPOUNDS }\end{array}$} \\
\hline & \multicolumn{5}{|c|}{ Inhibition (\%) } \\
\hline $5 \mathbf{a}$ & $57.86 \pm 0.70$ & $68.05 \pm 0.05$ & $73.93 \pm 0.13$ & $36.85 \pm 0.38$ & $52.70 \pm 0.63$ \\
\hline $5 b$ & $52.73 \pm 0.68$ & $57.30 \pm 0.70$ & $57.87 \pm 0.09$ & $57.14 \pm 0.29$ & $49.95 \pm 0.66$ \\
\hline $5 c$ & $69.72 \pm 0.45$ & $59.05 \pm 0.05$ & $64.07 \pm 0.00$ & $58.67 \pm 0.85$ & $54.94 \pm 0.63$ \\
\hline $5 e$ & $59.14 \pm 1.00$ & $53.75 \pm 0.45$ & $66.73 \pm 0.90$ & $62.90 \pm 0.10$ & $56.13 \pm 0.27$ \\
\hline $\mathbf{5 f}$ & $61.71 \pm 0.40$ & $43.60 \pm 0.30$ & $54.07 \pm 0.81$ & $39.33 \pm 1.00$ & $39.05 \pm 0.31$ \\
\hline Ciprofloxacin & $91.05 \pm 0.68$ & $92.32 \pm 0.42$ & $92.02 \pm 053$ & $91.44 \pm 0.64$ & $92.50 \pm 0.34$ \\
\hline
\end{tabular}

\begin{tabular}{cccccc}
\multicolumn{7}{c}{ MIC FOR ANTIBACTERIAL ACTIVITY OF SYNTHESIZED COMPOUNDS } \\
\hline \multirow{2}{*}{ Compounds } & \multicolumn{5}{c}{ Inhibition (\%) } \\
\cline { 2 - 6 } & Salmonella typhi $(-)$ & Escherichia coli $(-)$ & Pseudomonas aeruginosa $(-)$ & Staphylococcus aureus $(+)$ & Bacillus subtilis $(+)$ \\
\hline $\mathbf{5 a}$ & $15.98 \pm 0.45$ & $9.90 \pm 0.13$ & $18.99 \pm 0.10$ & - & $9.56 \pm 0.78$ \\
$\mathbf{5 b}$ & $14.39 \pm 0.31$ & $17.54 \pm 0.09$ & $17.61 \pm 0.24$ & $14.59 \pm 0.62$ & $10.55 \pm 0.52$ \\
$\mathbf{5 c}$ & $12.56 \pm 0.87$ & - & - & - & $17.78 \pm 0.05$ \\
$\mathbf{5 d}$ & $11.62 \pm 0.49$ & $19.12 \pm 0.89$ & - & - & $12.98 \pm 0.54$ \\
$\mathbf{5 e}$ & $9.90 \pm 0.12$ & $14.97 \pm 0.56$ & $17.89 \pm 0.12$ & $14.69 \pm 0.76$ & $11.67 \pm 0.43$ \\
$\mathbf{5 f}$ & $17.43 \pm 0.33$ & $15.67 \pm 0.34$ & - & $7.78 \pm 0.95$ & $16.42 \pm 0.12$ \\
Ciprofloxacin & $7.45 \pm 0.58$ & $7.16 \pm 0.58$ & $7.29 \pm 0.90$ & $7.80 \pm 0.19$ & $7.14 \pm 0.18$ \\
\hline
\end{tabular}

ciprofloxacin was $7.16 \pm 0.58 \mu \mathrm{M}$. The compound $\mathbf{5 f}$ showed moderate inhibition of all the microbes except Pseudomonas aeruginosa. The compound $\mathbf{5 d}$ was active against three strains Salmonella typhi, Escherichia coli and Bacillus subtilis; whereas compound $\mathbf{5 c}$ was active against only two strains, which are Salmonella typhi and Bacillus subtilis. If we take the antibacterial spectrum of these compounds into consideration, the compounds will have decreasing spectrum of antibacterial activity as $\mathbf{5 e}>\mathbf{5 b}>\mathbf{5 a}>\mathbf{5} \mathbf{f}>\mathbf{5} \mathbf{d}>\mathbf{5} \mathbf{c}$.

Compound $\mathbf{5 b}$ bearing chlorinated aliphatic substituent was active against all bacterial strains under study but compound 5c bearing brominated aliphatic substituent showed activity only against two bacterial strains. The results indicated that chloro group on aliphatic substituent had increased the spectrum of antibacterial activity of the synthesized. The antibacterial activity of compounds $\mathbf{5} \mathbf{d}$ and $\mathbf{5 e}$ indicated that the compounds bearing phenyl ring attached to smaller aliphatic chain were broad spectrum antibacterial agents.

\section{Conclusion}

The anticipated structures of compounds under study are supported and confirmed by spectroscopic data. Even though these compounds did not good enzyme inhibitors they are potent antibacterial agents. So these compounds, especially $\mathbf{5 e}$ and $\mathbf{5 b}$ can be considered for drug discovery and development program.

\section{ACKNOWLEDGEMENTS}

The authors are thankful to Higher Education Commission (HEC) of Pakistan for financial assistance. The authors are also thankful to Ministry of Higher Education (MOHE) under (FRGS) with sponsorship reference numbers FRGS/1/2016/ TK10/UITM/02/3 and Universiti Teknologi MARA for the financial support under the reference number 600-RMI/FRGS 5/3 (0119/2016).

\section{REFERENCES}

1. C.S. de-Oliveira, B.F. Lira, J.M. Barbosa-Filho, J.G.F. Lorenzo and P.F. de-Athayde-Filho, Molecules, 17, 10192 (2012); https://doi.org/10.3390/molecules 170910192.

2. K. Al-Othman, M. Abdulla, B. Jeppsson, H. Asakawa, U. Srinivas and S. Bengmark, Acta Pharm., 59, 223 (2009); https://doi.org/10.1111/j.1600-0773.1986.tb02749.x.

3. S.V. Bhandari, K.G. Bothara, M.K. Raut, A.A. Patil, A.P. Sarkate and V.J. Mokale, Bioorg. Med. Chem. Lett., 16, 1822 (2008); https://doi.org/10.1016/j.bmc.2007.11.014.

4. G.A. Idrees, O.M. Aly, G.D. Abuo-Rahma and M.F. Radwan, Eur. J. Med. Chem., 44, 3973 (2009); https://doi.org/10.1016/j.ejmech.2009.04.026

5. V. Padmavathi, G. Sudhakar Reddy, A. Padmaja, P. Kondaiah and AliShazia, Eur. J. Med. Chem., 44, 2106 (2009); https://doi.org/10.1016/j.ejmech.2008.10.012.

6. H. Kumar, S.A. Javed, S.A. Khan and M. Amir, Eur. J. Med. Chem., 43, 2688 (2008); https://doi.org/10.1016/j.ejmech.2008.01.039.

7. M.M. Burbuliene, V. Jakubkiene, G. Mekuskiene, E. Udrenaite, R. Smicius and P. Vainilavicius, Farmaco, 59, 767 (2004); https://doi.org/10.1016/j.farmac.2004.05.007.

8. G.R. Bankar, G.K. Nampurath, P.G. Nayak and S. Bhattacharya, Chem. Biol. Interact., 183, 327 (2010); https://doi.org/10.1016/i.cbi.2009.11.001.

9. Y. Shahar-Mohammad and A.W. Mohd, Acta Pol. Pharm., 66, 393 (2007).

10. M. Akhter, A. Husain, B. Azad and M. Ajmal, Eur. J. Med. Chem., 44, 2372 (2009);

https://doi.org/10.1016/j.ejmech.2008.09.005

11. O. Prakash, M. Kumar, R. Kumar, C. Sharma and K.R. Aneja, Eur. J. Med. Chem., 45, 4252 (2010); https://doi.org/10.1016/j.ejmech.2010.06.023.

12. K. Matsumoto, Y. Kawamura, Y. Yasuda, T. Tanimoto, K. Matsumoto, T. Yoshida and J. Shoji, J. Antibiot. (Tokyo), 42, 1465 (1989); https://doi.org/10.7164/antibiotics.42.1465.

13. A.A. Kadi, N.R. El-Brollosy, O.A. Al-Deeb, E.E. Habib, T.M. Ibrahim and A.A. El-Emam, Eur. J. Med. Chem., 42, 235 (2007); https://doi.org/10.1016/j.ejmech.2006.10.003.

14. A. Mumtaz, Saeed and I. Malik, J. Chem. Soc. Pak., 36, 852 (2014).

15. G.L. Ellman, K.D. Courtney, V. Andres Jr. and R.M. Featherstone, Biochem. Pharmacol., 7, 88 (1961); https://doi.org/10.1016/0006-2952(61)90145-9.

16. P. Chapdelaine, R.R. Tremblay and J.Y. Dube, Clin. Chem., 24, 208 (1978).

17. M. Kaspady, V.K. Narayanaswamy, M. Raju and G.K. Rao, Lett. Drug Des. Discov., 6, 21 (2009); https://doi.org/10.2174/157018009787158481. 